\title{
ÜBERSETZUNGSPROBLEME UND LÖSUNGSSTRATEGIEN BEI MUTTERSPRACHLICHEN UND NICHTMUTTERSPRACHLICHEN ÜBERSETZUNGEN INS DEUTSCHE
}

\author{
CHRISTOF HEINZ, BĚLA MICHÁLKOVÁ
}

\begin{abstract}
Translation strategies in native and non-native translations from Czech to German

The paper discusses the role of directionality in translations from Czech to German language. Its main purpose is to detect differences in the translation process between native and non-native translations, with a special focus on the translation strategies chosen. The empiric part consists of an investigation of a sample from students translations, produced during practical lessons on translation of non-literary texts into German at Charles University. Some of the examples discussed in the paper show differences between native and non-native translations on three different linguistic levels: lexical, syntactic and pragmatic. While the lexical and syntactic level show a tendency towards inhibitation of L1 structures, the pragmatic level is more likely to transfer source language norms into the target text.
\end{abstract}

Keywords: translation problems; translation strategies; Czech-German translation; directionality, native / non-native translation; crosslinguistic influence; L1-/ L2-transfer; lexical, syntactic, pragmatic transfer

\section{Einleitung}

In diesem Beitrag wird die Frage der Direktionalität in Übersetzungen zwischen den Sprachen Tschechisch und Deutsch erörtert. Mit dem Begriff „Direktionalität“ (oder „Übersetzungsrichtung“) ist dabei die Frage gemeint, ob die Sprache des Zieltextes für die Übersetzerin ${ }^{1}$ die eigene Muttersprache darstellt (L1-Übersetzung) oder eine später erlernte Fremdsprache (L2-Übersetzung), vgl. die Definition in Beeby Lonsdale (2011). Häufig steht in Untersuchungen zum Faktor Direktionalität die Frage der Qualität des Translats im Fokus (Prunč 2009). In diesem Beitrag wird jedoch weniger das Resultat des Übersetzungsvorgangs als der Prozess selbst und hier vor allem die Wahl der Über-

1 In diesem Artikel werden abwechselnd die weibliche und die männliche Form gebraucht, gemeint sind aber jeweils beide Geschlechter. 
setzungsstrategie im Mittelpunkt stehen. Die Hypothese ist, dass sich L1- und L2-Übersetzung womöglich nicht nur in Bezug auf das Ergebnis, sondern auch im Hinblick auf die vom Übersetzer gewählte Strategie unterscheiden. Eine mögliche Bestätigung der Hypothese soll anhand einiger Beispiele aus der Praxis der tschechisch-deutschen Übersetzerausbildung aufgezeigt werden. Das hierfür herangezogene Korpus studentischer Übersetzungen aus dem Tschechischen ins Deutsche erlaubt es jedoch aufgrund der Unausgewogenheit seiner Zusammensetzung (v.a. des deutlichen Überwiegens nichtmuttersprachlicher Übersetzungen) nicht, hierzu bereits statistisch valide quantitative Aussagen zu treffen. Im Rahmen dieses Beitrags können jedoch zumindest erste Tendenzen angedeutet werden, die einen Ausblick auf weitere, systematischere Untersuchungen zu dieser Problematik erlauben. Zunächst jedoch sollen einleitend Spezifika der Direktionalität beim Sprachenpaar Deutsch-Tschechisch, grundlegende Überlegungen zum Begriff der Übersetzungsstrategie, sowie Fragen des muttersprachlichen Einflusses bei der L2-Übersetzung und die Problematik der Direktionalität bei bilingualen Übersetzerinnen thematisiert werden.

\subsection{Direktionalität beim Sprachenpaar Deutsch-Tschechisch}

Der Faktor Direktionalität ist insofern als universal zu betrachten, als sein Einfluss auf den Übersetzungsvorgang und sein Ergebnis prinzipiell bei jedem Sprachenpaar beobachtet und thematisiert werden kann. In Bezug auf das konkrete Sprachenpaar DeutschTschechisch treten hierbei jedoch womöglich einige Besonderheiten auf, die sich aus der Spezifik des Verhältnisses der beiden Sprachen zueinander ergeben. Diese deutsch-tschechischen Spezifika sollen hier kurz resümiert werden.

In der, oftmals noch vorwissenschaftlichen, Beschäftigung mit Übersetzung genießt die L2-Übersetzung (oft auch „Version“ oder „Hinübersetzung“ im Gegensatz zur „Herübersetzung “ in die Muttersprache) einen eher zweifelhaften Ruf. Lange Zeit war es gewissermaßen common sense, dass Übersetzerinnen und v.a. Dolmetscherinnen bevorzugt, wenn nicht ausschließlich, in ihre Muttersprache übersetzen sollten. Als Grund hierfür wird meist die vermeintlich höhere Qualität des Translats genannt, da Muttersprachlern ein höherer Grad an Kompetenz bei der Zieltextproduktion zugestanden wird. Zweifel an dieser Sicht wurden in den letzten Jahrzehnten jedoch mehrfach geäußert (vgl. etwa Prunč 2009): Sie berufen sich zum einen darauf, dass die vermeintlichen Qualitätsunterschiede einer wissenschaftlichen Prüfung oft nicht standhalten, da andere Faktoren - etwa der Grad an Professionalität des Übersetzers, die Art der zu übersetzenden Texte oder äußere Umstände wie Zeitdruck und die Verfügbarkeit von Hilfsmitteln einen weit größeren Einfluss auf die Resultate haben als der Faktor Direktionalität. Nicht zuletzt basiert die einseitig negative Sicht auf L2-Übersetzungen auch auf einer Überbewertung der Tätigkeit der Zieltextproduktion gegenüber der Ausgangstextrezeption, bei der wiederum die Kompetenz von L2-Übersetzern höher eingeschätzt werden müsste. Letztlich liegt der Unterschied zwischen L1- und L2-Übersetzung somit wohl weniger in der Frage von höherer oder niedriger Qualität als vielmehr in der Art der typischerweise zu erwartenden „Übersetzungsfehler“.

Entscheidend für die Rehabilitierung der L2-Übersetzung sind aber oft einfach praktische Überlegungen, wie etwa die Tatsache der Verfügbarkeit von L1-Übersetzern. Hier 
spielen natürlich soziolinguistische und sprachenpolitische Faktoren wie die Verbreitung von Fremdsprachenkenntnissen im jeweils anderen Land eine zentrale Rolle. Und hier lässt sich gerade im Sprachenpaar Tschechisch-Deutsch ein deutliches Ungleichgewicht konstatieren. So nimmt in Tschechien die Bedeutung des Deutschen als Fremdsprache zwar seit der Jahrtausendwende kontinuierlich ab, dennoch konnte sich Deutsch nach Englisch als am zweithäufigsten gelernte Fremdsprache mittlerweile stabilisieren. Heute wählen ca. 60 \% der Schülerinnen in Tschechien Deutsch als 2. Fremdsprache, vgl. etwa die letzte verfügbare Netzwerk-Deutsch-Umfrage für Tschechien (Auswärtiges Amt 2015). Demgegenüber ist die Bedeutung des Tschechischen im deutschen Sprachraum als marginal zu bezeichnen. Das Angebot für Tschechisch als Fremdsprache an Schulen ist auf die bayerischen und sächsischen Grenzregionen - und auch dort meist auf den Wahlbereich - beschränkt. Berücksichtigt man dies, so ist es möglich, das Tschechische, obwohl sein Verbreitungsgebiet geographisch an den deutschen Sprachraum grenzt, innerhalb Deutschlands als language of low diffusion (LLD) im Sinne von Whyatt Pavlović (2019) zu bezeichnen, die unter diesem Begriff auch Sprachen subsumieren, die nur selten von Nicht-Muttersprachlern als Fremdsprache gelernt werden: „The term 'language of low diffusion' is understood to include (...) also those (languages), (...), that are rarely learned by non-native speakers" (Whyatt -Pavlović 2019, 102).

Die Folge für die Verfügbarkeit von Übersetzern liegt auf der Hand: Während für die Übersetzungsrichtung Deutsch-Tschechisch ausreichend muttersprachliche Übersetzerinnen mit Deutschkenntnissen zur Verfügung stehen, ist es für die Übersetzungsrichtung Tschechisch-Deutsch oftmals schwierig, deutsche Muttersprachler mit ausreichenden Kenntnissen des Tschechischen zu akquirieren. Es ist daher in der Praxis bei weitem keine Seltenheit, dass für die Übersetzung aus dem Tschechischen auf L2-Übersetzerinnen zurückgegriffen wird (werden muss?). Diese demographische und sprachenpolitische Schieflage wirkt sich auch auf die Gestaltung von Curricula in der Übersetzerausbildung für dieses Sprachenpaar aus: Während die Übersetzung in die Fremdsprache Tschechisch in der Ausbildung deutscher Übersetzerinnen eine eher untergeordnete Rolle spielt, da der Bedarf an muttersprachlichen Übersetzern weitgehend gedeckt ist, ist umgekehrt für die Ausbildung tschechischer Übersetzer mit Zielsprache Deutsch die Übersetzung in die Fremdsprache ein zentrales Lernziel, da L2-Übersetzungen auch in ihrer zukünftigen beruflichen Praxis zum Alltag gehören dürften.

Die Überlegungen zur L2-Übersetzung in diesem Beitrag beziehen sich folgerichtig auch stets ausschließlich auf die Übersetzungsrichtung Tschechisch-Deutsch, so stammen auch sämtliche Beispiele zum Vergleich muttersprachlicher und nichtmuttersprachlicher Übersetzungsstrategien aus Lehrveranstaltungen zur Übersetzung in die Zielsprache Deutsch.

\subsection{Zum Begriff der Übersetzungsstrategie}

In der Ausbildung tschechischer künftiger Übersetzerinnen spielt folglich die Entwicklung von Kompetenzen zur L2-Übersetzung in die Zielsprache Deutsch eine wichtige Rolle. Die dabei zu entwickelnden Kompetenzen umfassen jedoch nicht nur die bloße Sprachbeherrschung, sondern ebenso die Fähigkeit zum zielgerichteten Einsatz von Übersetzungsstrategien. Eingangs wurde bereits die Hypothese erwähnt, dass sich 
auch die Wahl der Übersetzungsstrategie in Abhängigkeit von der Übersetzungsrichtung unterscheiden könnte. Hier soll nun der Begriff der Übersetzungsstrategie wie er in translationswissenschaftlichen Beschreibungen des Übersetzungsprozesses verwendet wird, näher eingegrenzt werden.

Schon bei Hönig (1988) werden zwei grundlegende Auffassungen von „Übersetzungsstrategie“ einander gegenüber gestellt: eine „pragmatisch-funktionale“ gegenüber einer „psycholinguistischen“. Nach der etwa in Hönig - Kußmaul (1982) vertretene Auffassung von Übersetzungsstrategie als „planmäßigen Vollzug pragmalinguistischer Instruktionen“ (Hönig 1988, 10) lassen sich Gegensatzpaare wie „instrumentelle/funktionale“ vs. „dokumentarische“ oder „adaptierende“ vs. „exotisierende“ Übersetzung unterscheiden, vgl. etwa die Einteilung der Übersetzungsstrategien in Nord (2009) bzw. Stolze (2011). Da die Wahl einer so verstandenen Übersetzungsstrategie jedoch in erster Linie von der Textfunktion des Ausgangstexts abhängt, steht nicht zu erwarten, dass der Status der Zielsprache als L1 oder L2, und somit der hier untersuchte Faktor Direktionalität, für die Wahl einer solchen Übersetzungsstrategie überhaupt einen relevanten Faktor darstellt. Versteht man also unter „Übersetzungsstrategie“ eine bewusst getroffene Entscheidung der Übersetzerin zur Bewältigung der spezifischen kommunikativen Funktionen des Ausgangstextes, so ist davon auszugehen, dass diese unabhängig von Ziel- und Ausgangssprache und deren Status getroffen wird.

Anders sieht dies jedoch bei den psycholinguistisch orientierten Auffassungen von Übersetzungsstrategie aus: Diese legen den Fokus stärker auf den eigentlichen Übersetzungsprozess und untersuchen „welche mentalen Operationen beim Übersetzen überhaupt ablaufen“. Die Wahl einer so verstandenen Übersetzungsstrategie kann sehr wohl vom Status der Ausgangs- oder Zielsprache abhängen. So steht zu vermuten, dass L1-Übersetzer vorwiegend Strategien zum Einsatz bringen, die das korrekte Verständnis des Ausgangstextes ermöglichen, während L2-Übersetzerinnen sich viel stärker auf Strategien der Zieltextproduktion konzentrieren werden. Rezeptions- vs. Produktionsstrategien und ihre Relevanz für den Übersetzungsprozess lassen sich also vermutlich durchaus mit dem Faktor Direktionalität in Verbindung bringen. Im Gegensatz zu den pragmatisch verstandenen sind psycholinguistische Übersetzungsstrategien darüber hinaus auch nicht vollständig der bewussten Kontrolle unterworfen, so dass unbewusste Einflussfaktoren bei den Übersetzungsstrategien eine Rolle spielen können. Daher lassen sich für die psycholinguistisch orientierten Auffassungen von Übersetzungsstrategie auch Ergebnisse der Forschungen zum zwischensprachlichen Einfluss (crosslinguistic influence, CLI) aus dem Bereich der Spracherwerbsforschung für die übersetzungswissenschaftliche Fragestellung der Rolle der Übersetzungsrichtung für die Wahl der Strategie nutzbar machen. Für die Zwecke der eingangs skizzierten Forschungsfrage kann also sinnvollerweise nur von einem psycholinguistisch orientierten Verständnis von „Übersetzungsstrategie“ ausgegangen werden.

\subsection{Spracheneinfluss und Bilingualität bei der L2-Übersetzung}

Bei einer auf den Übersetzungsprozess fokussierenden Auffassung von Übersetzungsstrategien steht daher die Frage des zwischensprachlichen Einflusses mit der Frage der 
L2-Übersetzung in einem engen Zusammenhang, insbesondere die Frage, welchen Einfluss die L1 der Übersetzerinnen auf das Translat ausübt.

$\mathrm{Zu}$ erwarten ist hier, dass L1-Einfluss sich im L2-Translat in erster Linie durch Reproduktion von L1-Strukturen nachweisen lässt, die jedoch zielsprachlichen (grammatischen, lexikalischen, stilistischen) Normen widersprechen. Forschungen zu CLI im Fremdsprachenerwerb zeigen jedoch zweierlei: Zum einen kann neben der L1 auch der Einfluss aus anderen Sprachen (vorher erlernten Fremdsprachen) prinzipiell möglich sein. Dies gilt bei tschechischen Übersetzern umso mehr, als für sie das Deutsche häufig die zweite Fremdsprache nach Englisch darstellt, in der Erwerbsreihenfolge also als L3 bezeichnet werden müsste. Zudem wird die L2 Englisch gegenüber der L1 Tschechisch häufig als typologisch „näher“ am Deutschen konzeptualisiert, was die Wahrscheinlichkeit von Transfer aus der L2 weiter erhöht.

Außerdem ist neben der einfachen Reproduktion von L1- oder L2-Strukturen auch mit indirektem Einfluss auf den Zieltext zu rechnen (Jarvis - Pavlenko 2008). Dieser drückt sich dann nicht unmittelbar in Reproduktion, sondern in viel weniger leicht zu erfassenden Phänomenen Hyperkorrektismen oder Vermeidungsstrategien aus, die auf den ersten Blick nicht auf den Einfluss der L1 (oder L2) verweisen. Als hyperkorrekt können Fälle von Normverletzungen klassifiziert werden, die nicht mit entsprechenden Normen der L1 der Übersetzerinnen übereinstimmen, sondern Ergebnis einer Verallgemeinerung von zielsprachlichen Regeln und eine Missachtung von Beschränkungen für deren Anwendung darstellen. Demgegenüber sind als Ergebnisse von Vermeidungsstrategien Fälle zu beschreiben, in denen bestimmte, als „schwierig“ empfundene, zielsprachliche Strukturen gezielt vermieden, andere Strukturen hingegen bevorzugt werden. Das Ergebnis von Vermeidung besteht also nicht in tatsächlichen Normverletzungen, sondern eher in einer Abweichung von der erwarteten Frequenz im Zieltext, in dem bestimmte Konstruktionen über-, andere unterrepräsentiert sind. Dies kann sich wiederum auf den stilistischen Gesamtcharakter des Zieltextes negativ auswirken.

Schließlich soll noch kurz auf die Problematik der Direktionalität bei bilingualen Übersetzern, bei denen beide beteiligte Sprachen den Status einer L1 haben, eingegangen werden. Die Frage stellt sich, ob bei bilingualen Übersetzerinnen überhaupt von Direktionalität gesprochen werden kann, wenn keine der beiden Übersetzungsrichtungen als L2-Übersetzung, sondern als Übersetzung von einer L1 in die andere gelten kann. Gerade für das Sprachenpaar Deutsch-Tschechisch ist die Frage relevant, da gerade bilinguale Sprecher eine der größten Zielgruppen der Übersetzerausbildung darstellen. Jedoch stehen auch bei Bilingualen selten beide Sprachen in einem ausgewogenen Verhältnis. Meist wird im Laufe der sprachlichen Sozialisation eine der beiden Sprachen dominant, meist die Bildungssprache (Umgebungssprache), in der auch Literalität (literacy) erworben wird. Bei Bilingualen ist daher häufig die Entwicklung eines Diglossie-Verhältnisses zu beobachten, in der die Bildungssprache die Rolle der H-Variety einnimmt, in der sie über ein größeres Ausdrucksspektrum, eine sichere Beherrschung der Standnormen, sowie eine größere stilistische Bandbreite verfügen. Die L-Variety wird dagegen oft nur im familiären Umfeld verwendet, deren stilistisches Spektrum oft auf die Umgangssprache beschränkt ist, und in der die Kenntnis standardsprachlicher Normen nicht vorhanden oder zumindest nicht gefestigt ist. Statt von L1- und L2-Übersetzung könnte man daher bei Bilingualen vielleicht eher von H-Variety- und L-Variety-Übersetzung 
sprechen. Inwieweit sich L2-Übersetzung und Übersetzung in die L-Variety ähneln, wäre dann noch einmal ein ganz eigenes Untersuchungsgebiet.

\section{Praktischer Teil: Beispiele aus der Praxis der Übersetzerausbildung}

Die Überlegungen im ersten Teil sollen nun mit Beispielen aus der Praxis der Übersetzerausbildung illustriert werden. Die im Folgenden angeführten Beispiele entstammen einem Lerner-Korpus, das aus studentischen Übersetzungen in die Zielsprache Deutsch (Übungsaufgaben, Prüfungstexten) zusammengestellt wurde, die ich im Rahmen meiner Tätigkeit als Lektor am Institut für Translatologie der Karls-Universität Prag im Zeitraum von 2014 bis 2019 in Lehrveranstaltungen zur Übersetzung nichtliterarischer, z.T. fachsprachlicher Texte ins Deutsche sammeln konnte.

\subsection{Beispiele für Spracheneinfluss auf verschiedenen Ebenen}

Die ausgewählten Beispiele sollen stellvertretend für Übersetzungsstrategien auf 3 linguistischen Ebenen stehen: für Strategien bei der Wahl eines lexikalischen Äquivalents, für Strategien bei der Wahl einer grammatischen (syntaktischen) Konstruktion, sowie für Strategien bei der Wahl einer pragmatischen Komponente (hier: einer Anredeform). Der Vergleich von L1-/L2-Übersetzungen, die auf den jeweiligen Ebenen unterschiedliche Ergebnisse aufweisen, soll Rückschlüsse auf die unterschiedlichen gewählten Strategien zulassen.

\section{a) Im lexikalischen Bereich: Vermeidung von Anglizismen}

Als Beispiel soll die Wiedergabe des tschechischen služby in einem Werbetext des Internet-Anbieters UPC dienen. Während L2-Übersetzerinnen hier als Äquivalent am häufigsten „Dienstleistungen“ wählen (nur selten die wortwörtliche Wiedergabe mit „Dienste“), greifen L1-Übersetzer häufig auf den Anglizismus „Services“, auch in Zusammensetzungen wie „Serviceleistungen“, daneben aber auch zu lexikalisch weiter entfernten Lösungen wie „Angebot“, „Sortiment“ zurück. Dies deutet zum einen auf die Vermeidungsstrategie bei L2-Übersetzerinnen hin, die hier den Einfluss des Englischen als erster Fremdsprache zu unterdrücken versuchen. Die Tendenz zur Vermeidung von Anglizismen ist v.a. bei Fachtexten mit wirtschaftlicher Thematik häufig zu beobachten, in denen Anglizismen im Deutschen sehr verbreitet sind. Zum anderen deutet die größere Variabilität der lexikalischen Äquivalente auf ein breiteres Spektrum an möglichen Lösungen hin, die L1-Übersetzern zur Verfügung stellen, während bei L2-Übersetzerinnen die Tendenz zur Bevorzugung einer einmal gefundenen Lösung vorzuherrschen scheint.

\section{b) Im syntaktischen Bereich: Bevorzugung von SVO-Strukturen}

Im syntaktischen Bereich kann in vielen L2-Übersetzungen eine Tendenz zur Bevorzugung von SVO-Strukturen beobachtet werden, auch wenn im tschechischen Ausgangstext andere Wortfolge (etwa OVS) und diese auch im deutschen Zieltext problemlos möglich ist. Im Tschechischen ist die Satzgliedfolge OVS, gerade in publizistischen 
und administrativen Texten im Vergleich zum Deutschen deutlich frequenter, so dass tatsächlich nicht in jedem Fall die AT-Wortfolge beibehalten werden kann. Dennoch lässt das Deutsche in vielen Fällen OVS-Strukturen problemlos zu, und es stellt sich die Frage, warum L2-Übersetzer die mögliche Beibehaltung der ausgangssprachlichen Konstruktion vermeiden und scheinbar ohne Not zu einer komplexen Änderung der Satzkonstruktion greifen. Hier kann zum einen Unsicherheit über die tatsächliche zielsprachliche Korrektheit der beibehaltenen Konstruktion vorliegen, die zur Vermeidung der AT-Konstruktion führt. Eine andere Frage ist, ob auch hier Einfluss des Englischen vorliegen könnte, der sich diesmal in der direkten Reproduktion von Strukturen manifestiert. L2-Übersetzungen übertragen hier also grammatische Regeln von einer vorher erlernten Fremdsprache auf eine andere. Die generelle Tendenz zur Vermeidung von L1- Strukturen im Zieltext wird hier also durch zwischensprachlichen Transfer von der L2 auf die L3 unterstützt. L1-Übersetzungen greifen hier im Vergleich zu L2-Übersetzungen viel häufiger auf Passivkonstruktionen zurück.

\section{c) Im pragmatischen Bereich: Reproduktion von L1-Normen}

Als Beispiel dient hier ein Flyer einer tschechischen Hochschule, der für einen Studiengang wirbt - und sich auch sprachlich gezielt an ein studentisches Zielpublikum richtet, um die Anmeldung zum Studium möglichst niederschwellig erscheinen zu lassen.

Die Frage der Wahl des Übersetzungsäquivalents liegt hier im pragmatischen Bereich, konkret in der Wahl der Anredeform (Siezen oder Duzen). Die Formen im Ausgangstext geben hier keinen Anhaltspunkt, da die 2. Person Plural im Tschechischen im Hinblick auf die Anrede ambig ist (Duzen mehrerer Personen oder Siezen). Die Wahl der Anredeform muss also von der Übersetzerin aufgrund rein pragmatischer Gegebenheiten gewählt werden. Der Ausgangstext legt hier aufgrund der deutlichen Informalität Duzen nahe, dennoch entscheiden sich L2-Übersetzer zu $100 \%$ für die formale Anrede. Bei L1-Übersetzerinnen halten sich Du und Sie dagegen die Waage. In diesem Fall kann also tatsächlich von einer direkten Übertragung von L1-Normen auf den Zieltext gesprochen werden.

\subsection{Diskussion der beschriebenen Beispiele}

Eine vorsichtige erste Auswertung der beschriebenen Beispiele lässt im lexikalischen und syntaktischen Bereich eine Tendenz zu indirektem Transfer erkennen, v.a. zu Vermeidungsstrategien. Die gewählte Übersetzungsstrategie besteht hier in einer bewussten „Verfremdung“ des Zieltextes und in einer gezielten Entfernung von ausgangssprachlichen Strukturen.

Dagegen lässt sich direkter Transfer, also die Reproduktion von muttersprachlichen Strukturen im Zieltext am ehesten im pragmatischen Bereich beobachten. Im Beispiel ließ sich anhand der Wahl der formellen Anredeform trotz deutlicher Hinweise auf eine informelle Kommunikationssituation im Ausgangstext, die Übertragung pragmatischer Regeln aus der L1 in die Zielsprache nachweisen. Grund hierfür könnte sein, dass pragmatische Normen in der eigenen L1 für Übersetzerinnen weniger salient sind als Unterschiede in der sprachlichen Struktur. Eine Rolle kann auch die unbewusste Annahme universaler Gültigkeit pragmatischer Regeln spielen. 


\section{Ausblick: Weitere Untersuchungen zu diesem Problemkomplex}

Uns ist klar, dass die oben diskutierten Ergebnisse nichts mehr als erste Beobachtungen darstellen können, die mit gebotener Vorsicht betrachtet werden sollten. Vor allem ist uns die Unzulänglichkeit des zur Beispielsuche verwendeten Korpus bewusst. Dieses weist einige Nachteile auf, unter denen die Unausgewogenheit von L1- und L2-Übersetzungen (L2-Übersetzungen überwiegen bei weitem, L1-Übersetzungen, meist Arbeiten deutsch muttersprachlicher Austausch- und Erasmusstudierender, sind im Korpus dagegen deutlich unterrepräsentiert) sowie die mangelnde Vergleichbarkeit in Hinblick auf Bearbeitungszeit und Verwendung von Hilfsmitteln (viele der untersuchten Zieltexte waren Hausaufgaben) nur die auffälligsten sind. Auch ist natürlich ein Einfluss der Instruktion nicht auszuschließen, da die Übersetzungen im Rahmen einer Lehrveranstaltung entstanden sind, die gezielt sprachliche und strategische Kompetenzen vermitteln sollte. Wirklich statistisch aussagekräftige Ergebnisse können daher auf Basis der besprochenen Beispiele nicht erwartet werden. Ein Desiderat wäre daher die Erstellung eines ausgewogeneren Samples, das über die gleiche Anzahl von L1- und L2-Übersetzungen, die wiederum unter standardisierten Bedingungen, was Textlänge, Hilfsmittel und Bearbeitungszeit betrifft, angefertigt werden sollten.

Dennoch können erste Ergebnisse u.U. Hinweise auf lohnende künftige Untersuchungen bilden. Diese könnten auf einer Triangulierung der Methoden basieren: dabei kann die Beobachtung von realen Abweichungen in L1- und L2-Übersetzungen zur Identifizierung von möglichen Problemfeldern und Untersuchungsgegenständen dienen; eine quantitative Untersuchung unter standardisierten Bedingungen könnte statistisch valide Ergebnisse zur Verteilung der Abweichungen liefern; und schließlich könnten ergänzende qualitative Untersuchungen (etwa in Form von Lautdenkprotokollen) womöglich Antwort auf die Frage geben, ob hinter den beobachtbaren statistisch relevanten Unterschieden tatsächlich auch unterschiedliche Strategien von L1- und L2-Übersetzerinnen stehen.

Wir hoffen, mit diesem Beitrag zumindest einen ersten Schritt in diese Richtung unternommen und womöglich Anregungen für künftige Forschungsvorhaben im Bereich der deutsch-tschechischen Übersetzungswissenschaft gegeben zu haben.

\section{LITERATUR}

Auswärtiges Amt: Deutsch als Fremdsprache weltweit. Datenerhebung 2015. Online unter: https://www .dw.com/downloads/29827615/statistik-2015-deutschlerner-weltweit.pdf [06. 03. 2020].

Beeby Lonsdale, Allison: Directionality, in: Routledge Encyclopedia of translation studies, ed. Mona Baker - Gabriela Saldanha, second edition, London/New York: Routledge 2011, S. 84-88.

Chesterman, Andrew: Memes of Translation. The Spread of Ideas in Translation Theory, Amsterdam/ Philadelphia: John Benjamins 2000.

Feldmeier, Beate: Anrede im Sprachkontakt. Reflexion und Gebrauch von Anredestrategien durch tschechische Migranten im deutschsprachigen Umfeld, München: Sagner 2014.

Hönig, Hans G. - Kußmaul, Paul: Strategie der Übersetzung. Ein Lehr- und Arbeitsbuch, Tübingen: Narr 1982. 
Hönig, Hans G.: Wissen Übersetzer eigentlich, was sie tun?, in: Lebende Sprachen 1, 1988, S. 10-14.

Jarvis, Scott - Pavlenko, Aneta: Crosslinguistic influence in language and cognition, New York u.a.: Routledge 2008.

Klaudy, Kinga: Explicitation, in: Routledge Encyclopedia of translation studies, ed. Mona Baker, London/ New York: Routledge 1998, S. 80-84.

Levý, Jiří: Bude literárni věda exaktní vědou?, Praha: Čs. spisovatel 1971.

Nord, Christiane: Lernziel: Professionelles Übersetzen Spanisch-Deutsch. Ein Einführungskurs in 15 Lektionen, Wilhelmsfeld: Egert 2001.

Nord, Christiane: Textanalyse und Übersetzen. Theoretische Grundlagen, Methode und didaktische Anwendung einer übersetzungsrelevanten Textanalyse, Tübingen: Groos 2009.

Prunč, Erich: Translation in die Nicht-Muttersprache und Translationskultur, in: Translation into Non-Mother Tongues. In Professional Practice and Training, ed. Meta Grosman et al., Tübingen: Stauffenburg 2009, S. 5-20.

Snell-Hornby, Mary et al.: Handbuch Translation, Tübingen: Stauffenburg 1999.

Stellmacher, Tilo: Dossier Funktionales Übersetzen, 2013. Online unter: http://www.carstensinner.de /Lehre/uebersetzungswissenschaft/dossiers2013/Dossier_Funktionales_Uebersetzen_Stellmacher.pdf [19. 10. 2019].

Stolze, Radegundis: Übersetzungstheorien. Eine Einführung, Tübingen: Narr 2011.

Sulikowski, Piotr: Zur Strategie und Technik in der Übersetzung. Typologie der Übersetzungsstrategien, 2007. Online unter: https://www.db-thueringen.de/servlets/MCRFileNodeServlet/dbt_derivate _00011523/SulikowskiStrategie.pdf [17. 10. 2019].

Svoboda Tomáš: Kapitoly $z$ prekladatelské praxe. Odborný překlad mezi němčinou a češtinou, Praha: FF UK 2012.

Štícha, František: Česko-německá srovnávací gramatika, Praha: Argo 2003.

Whyatt, Bogusława - Pavlović, Nataša: Languages of low diffusion and low resources. Translation research and training challenges, in: The Interpreter and Translator Trainer, 13:1, 2019, S. 102-103.

Wille, Lucyna: Semantische Figuren in der Übersetzung. Ein Spiel mit Wort und Werk, Marburg: Tectum 2003.

Wilss, Wolfram: Übersetzungswissenschaft. Probleme und Methoden, Stuttgart: Klett 1977.

\section{RESUMÉ}

Článek se zabývá direkcionalitou v překladech z češtiny do němčiny, ve středu zájmu stojí proces překladu, a především volba překladatelské strategie rodilých mluvčích (překlady do L1) ve srovnání se strategiemi nerodilých mluvčích (překlady do L2). V praktické části jsou použity př́íklady z praxe (na základě studentských překladů semináře překlad neliterárních textů na Ústavu translatologie FF UK z let 2014-2019). Vybrané př́klady demonstrují překladatelské problémy a strategie na lexikální, syntaktické a pragmatické rovině. $Z$ vyhodnocení popsaných prŕkladů v překladech do L2 vyplývá, že na lexikální a syntaktické rovině lze pozorovat tendenci cíleného vyhýbání se strukturám výchozího textu. Reprodukce struktur L1 do cílového textu je naopak nejvíce patrná v rámci pragmatické roviny.

\section{Dr. Christof Heinz}

Westslawische Sprachwissenschaft, Universität Leipzig

christof.heinz@uni-leipzig.de

Mgr. Bèla Michálková, M.A.

Wissenschaftliche Hilfskraft im DAAD-Projekt „Interkulturelle Kommunikation und Translation Tschechisch-Deutsch“, Universität Leipzig

Bela.Michalkova@seznam.cz 